\title{
Quantum Nature of the Hydrogen Bond from Ambient Conditions down to Ultra-low Temperatures
}

\author{
Christoph Schran ${ }^{1, \text { a) }}$ and Dominik Marx ${ }^{1}$ \\ Lehrstuhl für Theoretische Chemie, Ruhr-Universität Bochum, 44780 Bochum, \\ Germany
}

(Dated: March 2, 2022)

\begin{abstract}
Many experimental techniques such as tagging photodissociation and helium nanodroplet isolation spectroscopy operate at very low temperatures in order to investigate hydrogen bonding. To elucidate the differences between such ultra-cold and usual ambient conditions, different hydrogen bonded systems are studied systematically from $300 \mathrm{~K}$ down to about $1 \mathrm{~K}$ using path integral simulations that explicitly consider both, the quantum nature of the nuclei and thermal fluctuations. For that purpose, finite sized water clusters, specifically the water dimer and hexamer, protonated water clusters including the Zundel and Eigen complexes, as well as hexagonal ice as a condensed phase representative are compared directly as a function of temperature. While weaker hydrogen bonds, as present in the neutral systems, show distinct structural differences between ambient conditions and the ultra-cold regime, the stronger hydrogen bonds of the protonated water clusters are less perturbed by temperature compared to their quantum ground state. In all studied systems, the quantum delocalization of the nuclei is found to vary drastically with temperature. Interestingly, upon reaching temperatures of about $1 \mathrm{~K}$, the spatial quantum delocalization of the heavy oxygens approaches that of the protons for relatively weak spatial constraints, and even significantly exceeds the latter in case of the centered hydrogen bond in the Zundel complex. These findings are relevant for comparisons between experiments on hydrogen bonding carried out at ultra-cold versus ambient conditions as well as to understand quantum delocalization phenomena of nuclei by seamlessly extending our insights into noncovalent interactions down to ultra-low temperatures.
\end{abstract}

Keywords: Hydrogen Bonds, Ultra-low Temperatures, Nuclear Quantum Effects, Path Integral Simulations

\section{INTRODUCTION}

The hydrogen bond has been the focus of numerous experimental studies some of which operate at rather low temperatures. Especially finite sized clusters are often investigated by means of tagging photodissociation ${ }^{1-3}$ or helium nanodroplet isolation ${ }^{4-8}$ spectroscopy techniques at temperatures on the order of 10 or even 1 Kelvin. Such spectroscopic techniques have been very successful in order to study hydrogen bonding in neutral water clusters $^{9-13}$, proton transfer in $\mathrm{HCl}$-water clusters ${ }^{14,15}$, as well as the microsolvation of the proton by water ${ }^{16-21}$ under cryogenic conditions. But also condensed phase hydrogen bonded systems such as hexagonal ice have been studied down to temperatures of about $1 \mathrm{~K}$ for example by neutron defraction ${ }^{22}$. Other temperature studies of ice $\mathrm{I}_{\mathrm{h}}$ have revealed interesting transitions in the thermal expansion coefficient around $100 \mathrm{~K}^{23}$ and also below $70 \mathrm{~K}$, where ice $\mathrm{I}_{\mathrm{h}}$ features negative thermal expansion ${ }^{24,25}$.

While such conditions significantly suppress thermal fluctuations and therefore provide usually a clean picture of the hydrogen bond, the system of interest is predominantly in its ground state, which drastically increases the importance of nuclear quantum effects (NQEs). These quantum effects such as tunneling and zero point vibrations need to be incorporated in any realistic simulation

a) Electronic mail: Christoph.Schran@rub.de on this topic if quantitative or even qualitative conclusions are of interest. However, already at moderately low or even ambient temperatures, the classical description of the nuclei misses some important features, in particular when hydrogen bonds are involved ${ }^{26-36}$. In addition, estimating the influence of NQEs on hydrogen bonding is no trivial task, since the quantum nature of the nuclei is known to show competing effects ${ }^{37,38}$. On the one hand, a weakening of the hydrogen bond is caused by the delocalization of the shared proton along the direction perpendicular to the hydrogen bond. On the other hand, the covalent $\mathrm{OH}$ bond gets elongated due to anharmonicity, thus resulting in shorter and thereby stronger hydrogen bonds. The degree of compensation of these competing effects is in general hard to predict since the shape of protons in hydrogen bonds are known since long to depend on the intermolecular bond length ${ }^{31}$, and offers more surprises when studied systematically ${ }^{36}$.

Independently from these phenomena, NQEs are starting to play a vital role in the correct description of the system of interest when decreasing the temperature. At the same time, their inclusion in quantum simulations increases the computational cost dramatically when reaching temperatures as low as $1 \mathrm{~K}$. Only recently, the efficient study of hydrogen bonded systems in that ultra-cold regime has become feasible via colored noise thermosttating methods ${ }^{39,40}$. This enables us to systematically investigate temperature effects on hydrogen bonding seamlessly from ambient conditions to $1 \mathrm{~K}$ while explicitly including the quantum nature of the nuclei. 
Hence, different hydrogen bonded systems, in particular the water dimer and hexamer as well as the three smallest protonated water clusters, the Zundel cation $\left(\mathrm{H}_{5} \mathrm{O}_{2}{ }^{+}\right)$, the protonated water trimer $\left(\mathrm{H}_{7} \mathrm{O}_{3}{ }^{+}\right)$, and the Eigen cation $\left(\mathrm{H}_{9} \mathrm{O}_{4}{ }^{+}\right)$, serving as representative finite sized clusters, are compared in detail in this study to the hexagonal phase of ice I in order to systematically study hydrogen bonding as a function of temperature from ambient conditions down to about $1 \mathrm{~K}$. Using these quite different system classes, various hydrogen bond characteristics and strengths can be studied in detail. These range from rather long and thus weak hydrogen bonds in the neutral systems to asymmetric double well situations in the larger protonated water clusters to a centered hydrogen bond in the Zundel cation which is often called "ultra-strong". It is, therefore, expected that our results can be transferred to various other hydrogen bonded systems depending on their hydrogen bond stengths.

\section{COMPUTATIONAL DETAILS}

To study the temperature dependence of different hydrogen bonded systems down to $1.67 \mathrm{~K}$, path integral molecular dynamics simulations have been performed with the i-PI program ${ }^{41}$ and the CP2k program package $^{42,43}$. The potential energy surface of the cationic and thus strong hydrogen bonded clusters, from the Zundel cation $\left(\mathrm{H}_{5} \mathrm{O}_{2}{ }^{+}\right)$over the protonated water trimer $\left(\mathrm{H}_{7} \mathrm{O}_{3}{ }^{+}\right)$, to the Eigen cation $\left(\mathrm{H}_{9} \mathrm{O}_{4}{ }^{+}\right)$, was described using a recently introduced neural network potential (NNP) fitted to coupled cluster reference calculations ${ }^{44}$ that is used here to carry out molecular dynamics simulations via CP2k. This NNP, which describes all investigated protonated species on equal footing, has been shown to not only match the reference coupled cluster theory with very high precision, but is also able to accurately describe proton transfer in these protonated species. The interactions in the three neutral systems of choice, i.e. the water dimer, water hexamer and hexagonal ice $\mathrm{I}_{\mathrm{h}}$, were described by the q-TIP4P/F force field ${ }^{37}$, which has been parametrized for the purpose of path integral simulations of water, via calls to LAMMPS ${ }^{45}$. That water model, where nuclear quantum effects effectively included in the parameterization of the original TIP4P model have been explicitly eliminated, has been shown to reproduce many of the fascinating properties of liquid water and ice with sufficient agreement to experiment ${ }^{37,46-51}$. In addition, it allows for fast and uncomplicated exploration at different conditions, which makes it perfectly suited in the present case. Recall that this non-reactive water model does not allow one to describe water dissociation and thus proton transfer by construction, which is an excellent approximation for our neutral water systems, whereas the reactive NNP is mandatory to simulate the protonated water clusters being prone to proton transfer events. Application of this force field to the water hexamer was previously shown to provide slightly inadequate populations of individual isomers ${ }^{52}$. This is expected to be of minor importance in the present case, since the individual properties of the hydrogen bonds in the various isomers of the water hexamer are very similar, which has been explicitly tested.

The systems of interest were simulated at temperatures of $300,250,100,20,10$ and $1.67 \mathrm{~K}$ including the quantum nature of the nuclei in conjunction with PIGLET thermostatting ${ }^{53}$ which has been recently extended to and validated at ultra-low temperatures ${ }^{39}$. In order to reach convergence, the path integral was discretized using $P=6,8,16,64,128$, and 256 replica at $T=300,250$, $100,20,10$, and $1.67 \mathrm{~K}$, respectively. The convergence of these path integral discretizations has been validated explicitly in Ref. 40 for the prototypical hydrogen bond in the Zundel cation and was chosen accordingly. In case of the water hexamer, which features different stable isomers, the interconversion was explicitly sampled between 100 and $300 \mathrm{~K}$, while below $100 \mathrm{~K}$ the system remained in its starting configuration. Therefore, different isomers were chosen as the starting point below $100 \mathrm{~K}$. In the following, results below $100 \mathrm{~K}$ are exclusively shown for the water hexamer in an ordered hexagonal ring in order to allow for a direct comparison with the hexagonal phase of ice. It is noted that the temperature dependence of the other isomers below $100 \mathrm{~K}$ is very similar to the results presented in the following. All reported simulations were propagated for at least $125 \mathrm{ps}$ using a formal molecular dynamics timestep of 0.25 fs where 2 ps at the beginning of each simulation were discarded as equilibration.

The condensed phase simulations were carried out using proton disordered ice $I_{h}$ supercells with periodic boundary conditions including 96 water molecules. This setup has been adapted from the established initial conditions underlying Refs. 54,55 . It features two hydrogen ordered hexamer rings, while the positions of the other hydrogen atoms were chosen to minimize the box dipole moment according to the usual ice rules. The cell parameters $(3 a, 2 \sqrt{3} a, 2 c)$, where $a$ and $c$ are the standard hexagonal lattice parameters of ice $\mathrm{I}_{\mathrm{h}}$, were chosen according to Ref. 24,25 for each simulation temperature. It is noted that while this work has been in progress, more precise measurements of the temperature dependent lattice parameters of ice $I_{h}$ have become available ${ }^{22,23}$. However, for the purpose of this work we do not expect large differences on the reported properties.

In order to validate the $\mathrm{q}-\mathrm{TIP} 4 \mathrm{P} / \mathrm{F}$ force field description of the interactions in ice at ultra-low temperatures, ab initio PIMD simulations were performed at $1.67 \mathrm{~K}$. These simulations were carried out with the CP2k program package ${ }^{42,43}$. The electronic structure was solved on-the-fly via the Quickstep module ${ }^{56}$ using the RPBE exchange-correlation functional ${ }^{57}$ together with the D3 dispersion correction ${ }^{58}$ taking into account the two-body terms and applying zero damping. The charge density was represented on a grid up to a plane wave cutoff of 500 Ry. The TZV2P basis set together with Goedecker-Teter-Hutter pseudopotentials to replace the 
core electrons of the oxygen atoms ${ }^{59}$ was used for the description of the Kohn-Sham orbitals. The SCF cycles were converged to an error of $\epsilon_{\mathrm{SCF}}=10^{-7} \mathrm{Ha}$. This electronic structure setup has been shown to describe many properties of water in close agreement to experiment ${ }^{60-62}$. In total $20 \mathrm{ps}$ (after an equilibration period of 1.5 ps using a starting configuration obtained from a well-equilibrated q-TIP4P/F force field simulation) were generated for analyses where the path integral has been discretized in terms of 48 replicas in conjunction with the PIGLET algorithm ${ }^{39,53}$

All reported properties were evaluated for hydrogen bonded configurations, where the standard hydrogen bond criterion based on a donor-acceptor distance of $3.5 \AA$ and a hydrogen bond angle $\angle \mathrm{HOO}$ of $30^{\circ}$ has been applied $^{63}$. Other hydrogen bond criteria were explicitly tested but resulted only in minor differences with respect to the reported results in agreement with earlier systematic studies on this topic ${ }^{64}$.

In order to estimate the hydrogen bond strength in the various studied systems, we calculated the binding energy per water monomer of each system for the optimized minimum energy configurations. As usual, the binding energy was obtained as the difference between the bound system and the sum of the isolated components, where for the protonated clusters the system was dissected into one hydronium core and the remaining water molecules. This binding energy was afterwards normalized by the number of water molecules present in the individual systems.

\section{RESULTS AND DISCUSSION}

In order to both, motivate and illustrate the drastically changing relative importance of NQEs for hydrogen bonding when decreasing the temperature from ambient to ultra-cold conditions, we start by discussing the temperature dependence of the total position fluctuations $\left\langle\Delta x^{2}\right\rangle_{\text {tot }}$ of a hydrogen atom trapped in a $1 \mathrm{D}$-harmonic potential serving as a simple textbook example. These total fluctuations can be straight-forwardly decomposed into the respective quantum delocalization, $\left\langle\Delta x^{2}\right\rangle_{\mathrm{q}}$, and the purely classical position fluctuations $\left\langle\Delta x^{2}\right\rangle_{\mathrm{c}}$ as shown in Fig. 1. The quantum contribution is the difference between a purely classical description of the nuclei and the correct total fluctuations at the respective temperature. For the simple harmonic oscillator, the quantum delocalization $\left\langle\Delta x^{2}\right\rangle_{\mathrm{q}}$ ultimately converges to a constant value when the temperature is lowered, where it provides a measure of the harmonic zero point motion, whereas it decays to strictly zero in the high temperature (classical) limit as shown in Fig. 1. In contrast, the classical (thermal) fluctuations $\left\langle\Delta x^{2}\right\rangle_{\mathrm{c}}$ strictly vanish at zero temperature but increase linearly as a function of temperature, see Fig. 1 (note the logarithmic temperature scale). The most interesting regime is at intermediate temperatures where both, quantum and thermal fluctu-

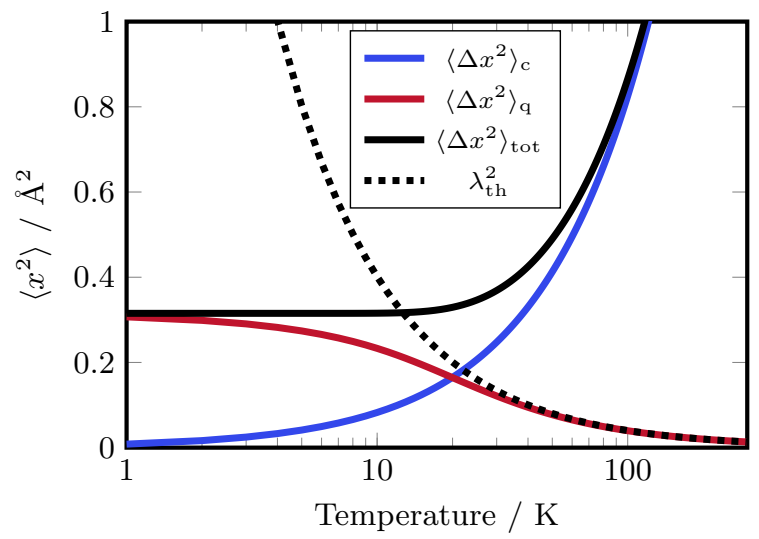

Figure 1. Comparison of the temperature dependent position fluctuations of a hydrogen atom trapped in a 1D-harmonic potential with a period of $2 \pi \cdot 100 \mathrm{fs}$. The total fluctuations $\left\langle\Delta x^{2}\right\rangle_{\text {tot }}$ are shown in black, the classical fluctuations $\left\langle\Delta x^{2}\right\rangle_{\mathrm{c}}$ in blue and the quantum fluctuations $\left\langle\Delta x^{2}\right\rangle_{\mathrm{q}}=\left\langle\Delta x^{2}\right\rangle_{\text {tot }}-$ $\left\langle\Delta x^{2}\right\rangle_{\mathrm{c}}$ in red, where $\langle\cdots\rangle_{\mathrm{c}}$ and $\langle\cdots\rangle_{\text {tot }}$ are the classical and quantum statistical Maxwell-Boltzmann (canonical) averages respectively, at the given temperature. The squared thermal wavelength $\lambda_{\text {th }}^{2}$ of a free hydrogen atom in one dimension is included for comparison as dotted line.

ations contribute significantly, as indicated in Fig. 1 by the crossing of the $\left\langle\Delta x^{2}\right\rangle_{\mathrm{q}}$ and $\left\langle\Delta x^{2}\right\rangle_{\mathrm{c}}$ curves, which can be used to define the so-called "crossover temperature" between quantum and classical behavior ${ }^{65}$.

Obviously, realistic molecular systems that include hydrogen bonds are governed by pronounced anharmonic covalent and noncovalent interactions, which adds complexity to the undergraduate textbook picture that is illustrated with the help of Fig. 1. These anharmonic effects together with the pronounced many-body nature of the underlying intermolecular interactions are the major reason for the rich characteristics of the hydrogen bond, ranging from weak and thus strongly asymmetric such non-covalent bonds with sizable proton transfer barriers to low-barrier situations to even centered or ultra-strong hydrogen bonding as for example all encountered during the phase transition from molecular ice VIII to ionic ice $\mathrm{X}^{66}$. Therefore, a systematic study is conducted in what follows in order to understand quantitatively how hydrogen bonding is influenced as a function of temperature down to the ultra-cold regime depending on the hydrogen bonding class in the aforementioned sense. In experiment only the total quantum solution is accessible, which is why we base our analysis purely on quantum simulations. For that purpose, strongly hydrogen bonded systems, namely the protonated water dimer (Zundel complex), trimer and tetramer (Eigen complex) as well as three weaker hydrogen bonded systems, being the water dimer and hexamer in vacuum and the hexagonal phase of ice $I$, ice $I_{h}$, have been selected to serve as representative cases. In these systems the gradual increase of hydrogen bonding and resulting cooperative effects ${ }^{67,68}$ can 
be studied in detail, while additionally granting access to general differences and similarities between finite clusters and the condensed phase as well as charged and neutral systems. Note that in our neutral systems the rather long hydrogen bonds lead to very high proton transfer barriers and thus greatly suppress proton transfer, while the protonated water clusters are much more prone to proton transfer events.

As illustrated based on the simple harmonic oscillator example the relative importance of the quantum nature of the nuclei increases when the temperature is lowered, but for most structural properties, convergence with temperature is found to be reached relatively fast. In order to investigate structural properties, the distribution functions of three main properties of the hydrogen bond for the different systems are compared in Fig. 2 at 250 and $1.67 \mathrm{~K}$. The chosen properties, namely the donoracceptor distance $r_{\mathrm{OO}}$, the proton sharing coordinate $\delta$, and the hydrogen bond angle $\angle_{\mathrm{HOO}}$, are ideally suited to characterize hydrogen bonding and all directly relate to the strength of the hydrogen bond. In general, shorter heavy atom distances, more symmetric sharing coordinates, and smaller hydrogen bond angles correlate well with stronger hydrogen bonding ${ }^{38,69-71}$, which allows us to order the six systems from top to bottom in that figure. To further quantify the hydrogen bond strength in the studied systems, we evaluated the binding energy per water molecule as described in Sec. II. The resulting rough estimate of the hydrogen bond strength is also included in Fig 2 (right vertical scale) and can be used to provide a comparison for other hydrogen bonded systems, not included in the present study, in order to judge their dependence on nuclear quantum effects depending on the temperature regime.

Let us first discuss in detail the heavy atom distance $r_{\mathrm{OO}}$ at the two temperatures. While for the water dimer at $1.67 \mathrm{~K}$ a relatively broad distribution with an average at about $2.85 \AA$ is found, this distribution is shifted towards smaller distances to an average of $\approx 2.80 \AA$ in the water hexamer and finally to about $2.75 \AA$ in the bulk where it is also much more narrow. When moving to the stronger hydrogen bonds of the protonated water clusters, the low temperature distributions become even sharper and are shifted to shorter $r_{\mathrm{OO}}$ distances. The distribution peaks at $2.56 \AA$ for the Eigen cation, while being moved to $2.49 \AA$ for the protonated water trimer and finally to $2.41 \AA$ for the Zundel cation. In case of the neutral finite clusters, increasing the temperature to $250 \mathrm{~K}$ causes distinct changes, more specifically broadening the distributions and shifting them to larger distances. In the condensed phase the temperature effect is less dominant, while it diminishes further when moving to the protonated water clusters. Note that the $r_{\mathrm{OO}}$ distributions of the Zundel cation are almost indistinguishable although the temperature difference is about $250 \mathrm{~K}$.

Secondly, the proton sharing coordinate $\delta$, defined as the difference between the covalent bond length and the noncovalent bond length (i.e. the hydrogen bond distance) $\delta=r_{\mathrm{OH}}-r_{\mathrm{H} \cdots \mathrm{O}}$, is analyzed to identify the degree of asymmetry in the hydrogen bond. This coordinate is systematically shifted towards a more symmetric hydrogen bond when comparing the water dimer, hexamer, ice, and the three protonated water clusters. This can be seen from the averages that decrease from $-0.95 \AA$ in the water dimer to $-0.90 \AA$ in the hexamer to $-0.83 \AA$ in ice. For the Eigen cation, the average of the distribution is around $-0.55 \AA$, which is shifted to $-0.40 \AA$ in the protonated water trimer and finally to zero for the Zundel cation, indicating a centered hydrogen bond. Again, the distributions of the neutral finite sized clusters are broader and shifted to even more asymmetric values at $250 \mathrm{~K}$, while very similar distributions are obtained for ice and the three protonated water clusters at both temperatures. As already found for the hydrogen bond length, the distributions of the Zundel cation are essentially indistinguishable at both temperatures.

Finally, the hydrogen bond angle $\angle_{\mathrm{HOO}}$ is selected to characterize the degree of deviation from collinearity in the hydrogen bond, which is the third proxy that correlates with the strength of hydrogen bonds. Here, the distributions of the neutral finite clusters peak at slightly larger angles than in the condensed phase and also feature a longer tail towards larger angles, thus implying weaker hydrogen bonding in the neutral finite systems. In case of the protonated clusters, the distributions are shifted further to smaller angles when going from the Eigen to the Zundel cation. As before, temperature does essentially not affect the respective distribution in ice and the protonated water clusters, whereas it shifts it to larger angles in the neutral finite clusters by about $2-3^{\circ}$. Thus, temperature weakens the hydrogen bond further in the water dimer and hexamer compared to ice.

Overall, these results reveal clear trends in hydrogen bonding of the three neutral systems when the coordination of the individual water molecules is increased from finite sized clusters to the condensed phase. Due to the higher degree of hydrogen bonding and the resulting cooperative effects ${ }^{68}$, overall shorter, more symmetric and more linear, and therefore stronger hydrogen bonds are observed in the condensed phase. Interestingly, an opposite cooperative effect is observed when analyzing the protonated water clusters (while keeping in mind their different hydrogen bonding topology). All structural properties indicate for these systems and topologies that the addition of further water molecules is weakening the hydrogen bond, since the donor-acceptor distance is increased, the sharing coordinate moves to more asymmetric values and the hydrogen bond angle is progressively increased. This can be explained by the larger degree of charge delocalization when more water molecules solvate the protonic defect.

In addition, this comparison of different structural properties of the hydrogen bond reveals that the temperature effects from 250 down to $1.67 \mathrm{~K}$ have only a minor impact on the structural properties for the stronger hy- 


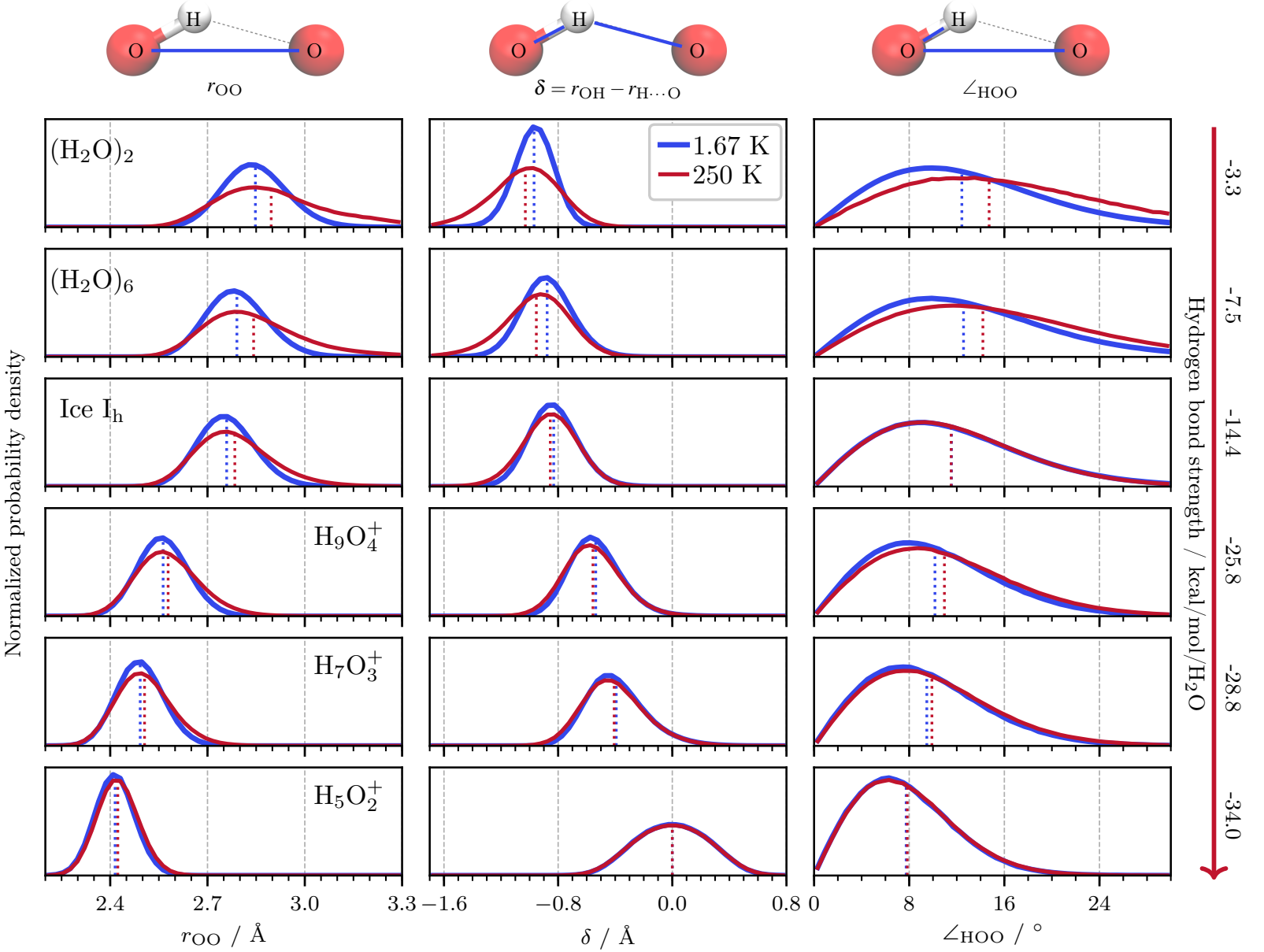

Figure 2. Normalized probability distributions of the heavy atom donor-acceptor distance $r_{\mathrm{OO}}$ (left), the proton sharing coordinate $\delta$ (middle) defined as $\delta=r_{\mathrm{OH}}-r_{\mathrm{H} \ldots \mathrm{O}}$, and the hydrogen bond angle $\angle \mathrm{HOO}$ (right) from PIMD simulations at $1.67 \mathrm{~K}$ (blue) and $250 \mathrm{~K}$ (red) for the water dimer $\left(\left(\mathrm{H}_{2} \mathrm{O}\right)_{2}\right)$, water hexamer $\left(\left(\mathrm{H}_{2} \mathrm{O}\right)_{6}\right)$, ice $\mathrm{I}_{\mathrm{h}}$, Eigen cation $\left(\mathrm{H}_{9} \mathrm{O}_{4}^{+}\right)$, protonated water trimer $\left(\mathrm{H}_{7} \mathrm{O}_{3}{ }^{+}\right)$, and Zundel cation $\left(\mathrm{H}_{5} \mathrm{O}_{2}{ }^{+}\right)$from top to bottom. The different systems were ordered by increasing hydrogen bond strength (cf. decreasing average $r_{\text {OO }}$ distance and $\angle$ HOO angle) from top to bottom according to the estimated hydrogen bond strength as described in Sec. II. The average of the individual distributions is marked with a vertical dotted line. Exclusively hydrogen bonded configurations are considered in this analysis based on the hydrogen bond criterion of Ref. 63.

drogen bonds, or even no impact in case of the strongest (centered) hydrogen bond as offered by the Zundel complex (thus confirming pioneering work ${ }^{26}$ ). This reveals that for the Zundel cation the main contribution to the structural properties of the hydrogen bond comes from the nuclear zero point energy even at a temperature as high as $250 \mathrm{~K}$. Still, temperature has a bigger impact on the larger protonated water clusters and ice, as seen from the detailed comparison of the distributions between 1.67 and $250 \mathrm{~K}$. Note that these observations are also in line with a recent spectroscopic investigation of the Eigen cation, for which only a very mild temperature dependence of the spectrum has been reported ${ }^{21}$. At the same time, the neutral finite clusters feature a systematic weakening of hydrogen bonding when increasing the temperature.

When the results for the different hydrogen bonded systems are compared directly, it can be concluded that minor temperature effects are observed for strong hydrogen bonds when moving from 1.67 to $250 \mathrm{~K}$, but weaker hydrogen bonds feature larger changes at ambient conditions compared to ground state dominated temperatures. This underlines that ultra-low temperature conditions are ideally suited to study strong hydrogen bonds, while for weaker hydrogen bonds the results need to be carefully transferred to ambient conditions.

Let us next analyze the contribution of NQEs on the quantum de/localization of the individual atoms participating in the hydrogen bond, i.e. oxygens and hydrogens. As introduced above for the harmonic oscillator, this property features a strong dependence on the temperature. Indeed, recent studies at ultra-low temperature have revealed an unexpected localization of the nuclear wavefunction in finite hydrogen bonded complexes ${ }^{72}$, es- 
pecially for the light hydrogen atoms participating in intermolecular interactions. This effect is even further increased due to helium-solute interactions ${ }^{73}$. At the same time, the heavy donor and acceptor atoms of these hydrogen bonded clusters are less affected and can even be more quantum delocalized than the much lighter protons in the hydrogen bond. Recall that the thermal de Broglie wavelength $\lambda_{\text {th }}$ of a free particle scales with $1 / \sqrt{M}$. From this consideration it is usually expected that protons are much more quantum delocalized than the heavier oxygen nuclei at the same temperature, which highlights the unexpected character of the previously discovered ${ }^{72,73}$ larger delocalization of the oxygen atoms in these hydrogen bonds at temperatures on the order of $1 \mathrm{~K}$. This so-called "interaction induced localization effect" on protons trapped in hydrogen bonds is far from being understood and needs further investigation as a function of temperature.

In order to systematically investigate such effects for the various systems of the present study the quantum delocalization of the oxygen atoms as well as the hydrogen atoms participating in hydrogen bonds are analyzed in detail. Quantum delocalization of nuclei at a given temperature can be conveniently separated from the total fluctuations in the path integral formalism by measuring the instantaneous spread of the path integral ring polymer with reference to its centroid position. The resulting averaged radius of gyration of the discretized path integral

$$
r_{\mathrm{g}}^{2}=\frac{1}{P} \sum_{s=1}^{P}\left\langle\left(\mathbf{R}_{s}-\mathbf{R}_{\mathrm{c}}\right)^{2}\right\rangle,
$$

is depicted as a function of temperature in Fig. 3 for the individual atoms. This property, if squared, is identical to the quantum fluctuations $\left\langle\Delta x^{2}\right\rangle_{\mathrm{q}}$ that were introduced above. In addition, in the case of a free particle, the radius of gyration is equivalent to the thermal de Broglie wavelength and thus gives access to the quantum delocalization of the interacting nuclei of interest at a certain temperature.

At $300 \mathrm{~K}$ the oxygen atoms feature only a relatively small average radius of gyration in all systems according to Fig. 3. This indicates that their quantum delocalization adds a rather small contribution to the classical description of the oxygen atoms. At the same temperature, the hydrogen atoms in all three systems are considerably more quantum delocalized. This not only highlights the importance of NQEs on the properties of the hydrogen bond already close to ambient conditions, but also confirms general expectations based on the stark differences of the thermal de Broglie wavelength of heavy versus light free particles. Together, these data support the well-known picture that heavy atoms, such as oxygen nuclei, behave essentially like classical point particles at ambient temperature, while the light protons are already affected by NQEs and thus "smeared out", which is independent of the particular system. Most interestingly, all chosen systems covering a broad range of hydrogen bond strengths feature essentially the same radius of gyration for the hydrogen and oxygen nuclei as seen by the respective horizontal dashed lines in Fig. 3.

As the temperature decreases, both the oxygen and hydrogen atoms in all systems become less localized, but a relatively constant offset between the hydrogen and oxygen atoms is initially conserved. However, for the three smallest finite clusters this offset starts decreasing at about $10 \mathrm{~K}$ to finally result in a slightly larger average radius of gyration of the oxygen nuclei compared to the protons at around $1 \mathrm{~K}$. This effect is most pronounced for the centered (or "ultra-strong") hydrogen bond present in the Zundel cation, $\mathrm{H}_{5} \mathrm{O}_{2}{ }^{+}$. In other words: The quantum delocalization of the heavy oxygen nuclei exceeds that of the light protons in this temperature regime as opposed to the usual scenario that is recovered at higher temperatures. Exactly this "interaction induced localization" of the protons in hydrogen bonds at ultra-low temperatures has first been described in Ref. 72 for various hydrogen bonded dimers - including even the heavier chlorine nucleus when $\mathrm{HCl}$ is involved in hydrogen bonding. It is important to note that this effect is not an artifact of the underlying force field description of the interactions as used previously ${ }^{72}$. Indeed, very similar results are obtained for the Zundel cation, $\mathrm{H}_{5} \mathrm{O}_{2}{ }^{+}$, and the protonated water trimer, $\mathrm{H}_{7} \mathrm{O}_{3}{ }^{+}$, on an essentially converged potential energy surface fitted to coupled cluster reference calculations ${ }^{44}$. The water hexamer and the Eigen cation reveal a similar temperature dependence, but the oxygen atoms remain more localized than the hydrogen atoms at the lowest considered temperature, although the difference between the two atoms is significantly descreased at $1 \mathrm{~K}$ compared to $250 \mathrm{~K}$; it is tempting to speculate that the crossover might set in at still lower temperatures.

In stark contrast to the finite sized systems, the difference in the quantum delocalization between oxygen and hydrogen atoms as provided by the radius of gyration remains almost constant over the whole temperature range for ice $I_{h}$ as seen in the right panel of Fig. 3. Note that also these force field results were validated by $a b i n i$ tio PIMD simulations using the RPBE-D3 density functional (see Sec. II for the details), providing a very similar delocalization at the lowest considered temperature (see the triangles in the right panel of Fig. 3). In addition, the extent of the quantum delocalization of both atoms is overall significantly smaller compared to the finite clusters at the lower temperatures and, at variance with the behavior found for these finite clusters, seems to flattens out. These results reveal that the "interaction induced localization effect" found for finite clusters in vacuum can not be transferred to ice. At the same time, almost the same quantum delocalization is found at higher temperatures for all systems, which clearly needs to be analyzed in more detail.

We now focus on an explanation for the different localization effects of the hydrogen bond in isolated clusters 


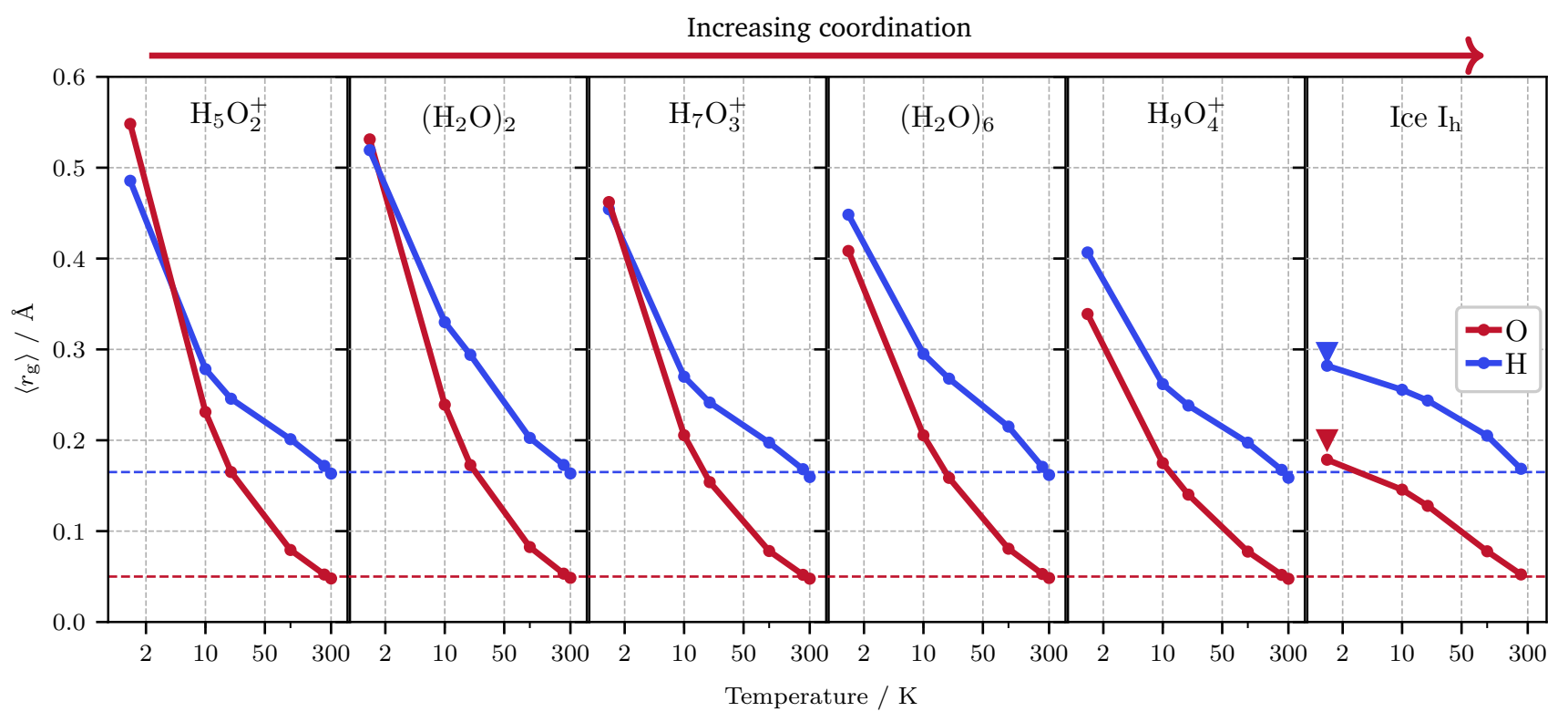

Figure 3. Temperature dependence of the quantum delocalization of the oxygen (red) and hydrogen atoms involved in hydrogen bonds (blue) measured by the averaged radius of gyration $\left\langle r_{\mathrm{g}}\right\rangle$ in the Zundel cation $\left(\mathrm{H}_{5} \mathrm{O}_{2}{ }^{+}\right)$, water dimer $\left(\left(\mathrm{H}_{2} \mathrm{O}\right)_{2}\right)$, protonated water trimer $\left(\mathrm{H}_{7} \mathrm{O}_{3}{ }^{+}\right)$, water hexamer $\left(\left(\mathrm{H}_{2} \mathrm{O}\right)_{6}\right)$, Eigen cation $\left(\mathrm{H}_{9} \mathrm{O}_{4}{ }^{+}\right)$, and ice $\mathrm{I}_{\mathrm{h}}$ from left to right. The different systems were ordered by increasing coordination from left to right. In order to highlight the similarities between the different systems, the average values of all systems at the highest considered temperatures are included as horizontal dashed lines for the oxygen and hydrogen atoms, respectively. Exclusively hydrogen bonded configurations are considered in this analysis. All results for the neutral systems are obtained using the q-TIP4P/F water force field, while the cationic systems were described by a highly accurate neural network potential (NNP) of coupled cluster accuracy (see text). For ice $\mathrm{I}_{\mathrm{h}}$, the results at the lowest temperature were validated using ab initio path integral simulations based on RPBE-D3 (see text) electronic structure (triangles).

versus hexagonal ice. Key to the understanding of the differences is the coordination of the individual hydrogen bond partners in the various systems. As indicated by the ordering in Fig. 3 this coordination gradually increases when moving to the larger systems. While the two dimers feature only a single hydrogen bond, every water molecule in the (cyclic) water hexamer participates in two hydrogen bonds, and the hydronium core of the Eigen cation forms three hydrogen bonds. Finally, in ice $\mathrm{I}_{\mathrm{h}}$ every water molecule donates and accepts two hydrogen bonds, resulting in a coordination number of four per molecule. The increase in the coordination clearly correlates with the overall size of the atoms at ultra-low temperatures and additionally with the crossover between oxygen and hydrogen atoms. As illustrated above using the simple harmonic oscillator model, the constraining potential of an atom shows the biggest impact at low temperatures, while at higher temperatures essentially the thermal wavelength of the respective free particles is recovered. This explains why the coordination modulates the size of the atoms mainly at low temperatures, while essentially the same radii of gyration are observed at ambient conditions. It can therefore be concluded that enhanced coordination of the atoms, which counteracts translational and rotational delocalization and constraints the quantum fluctuations of the atoms, modulates the "interaction induced localization effect" and entirely prevents it in the condensed phase.

Overall, the detailed analysis of quantum delocalization in the hydrogen bond reveals that the previously reported "interaction induced localization effect" can be found in finite sized clusters, whereas it is absent in the condensed phase. In addition, this difference is traced back to the coordination of the involved atoms. Given the systematic study of various systems, it is expected that these results are rather general for comparing finite sized clusters and condensed phase hydrogen bonded systems.

\section{CONCLUSIONS}

In summary, hydrogen bonding has been investigated in detail as a function of temperature, from ambient $(300 \mathrm{~K})$ down to ultra-cold $(\approx 1 \mathrm{~K})$ conditions, including nuclear quantum effects for various hydrogen bonded systems, in particular for the water dimer and hexamer as well as for protonated water clusters from the Zundel to the Eigen complex, and hexagonal ice $\mathrm{I}_{\mathrm{h}}$. Our analysis revealed minor temperature effects on structural properties of the hydrogen bond, specifically its length, angle and a/symmetry, for strongly hydrogen bonded systems, such as protonated water clusters including the Zundel complex, when moving from about $1 \mathrm{~K}$ up to ambient 
conditions. In stark contrast, weaker hydrogen bonds, as present in the investigated neutral systems and notably in the water dimer, were found to be significantly altered when moving from ground state dominated temperatures to ambient conditions, where mainly additional weakening of this noncovalent bond is observed. These systematic results unveil that ultra-low temperature experiments such as tagging photodissociation and helium nanodroplet isolation spectroscopy are ideally suited to study strong hydrogen bonds, while for weaker hydrogen bonds the results need to be carefully transferred to ambient conditions.

Furthermore, the quantum delocalization of the nuclei involved in hydrogen bonding was explored in detail to reveal considerable differences between finite clusters in vacuum and the condensed phase. In contrast to the condensed phase, the quantum delocalization of the heavier oxygen atoms was found to be comparable to, or even larger than that of the hydrogen atoms in systems with relatively weak spatial constraints. These differences were traced back to the coordination of the involved atoms which counteracts translational and rotational quantum delocalization. In addition, the coordination was shown to be the main driving force behind the intriguing larger localization of the lighter hydrogen atoms compared to the oxygen atoms as previously reported for hydrogen bonded clusters in superfluid helium nanodroplets based on quantum simulations in explicit bosonic helium.

\section{ACKNOWLEDGEMENTS}

It gives us great pleasure to thank Harald Forbert, Fabien Brieuc, and Felix Uhl for helpful discussions. This research is part of the Cluster of Excellence "RESOLV" (EXC 2033, ID 390677874) funded by the Deutsche Forschungsgemeinschaft, DFG. C.S. acknowledges partial financial support from the Studienstiftung des Deutschen Volkes as well as from the Verband der Chemischen Industrie. The computational resources were provided by HPC@ZEMOS, HPC-RESOLV, and BOVILAB@RUB.

\section{REFERENCES}

${ }^{1}$ S. Chakrabarty, M. Holz, E. K. Campbell, A. Banerjee, D. Gerlich, and J. P. Maier, J. Phys. Chem. Lett. 4, 4051 (2013).

${ }^{2}$ A. B. Wolk, C. M. Leavitt, E. Garand, and M. A. Johnson, Acc. Chem. Res. 47, 202 (2014).

${ }^{3}$ J. Roithová, A. Gray, E. Andris, J. Jašík, and D. Gerlich, Acc. Chem. Res. 49, 223 (2016).

${ }^{4}$ S. Goyal, D. L. Schutt, and G. Scoles, Phys. Rev. Lett. 69, 933 (1992).

${ }^{5}$ J. P. Toennies and A. F. Vilesov, Annu. Rev. Phys. Chem. 49, 1 (1998).

${ }^{6}$ F. Stienkemeier and A. F. Vilesov, J. Chem. Phys. 115, 10119 (2001).
${ }^{7}$ J. P. Toennies and A. F. Vilesov, Angew. Chem. Int. Ed. 43, 2622 (2004).

${ }^{8}$ F. Stienkemeier and K. K. Lehmann, J. Phys. B 39, R127 (2006). ${ }^{9}$ K. Liu, M. G. Brown, C. Carter, R. J. Saykally, J. K. Gregory, and D. C. Clary, Nature 381, 501 (1996).

${ }^{10}$ J. D. Cruzan, L. B. Braly, K. Liu, M. G. Brown, J. G. Loeser, and R. J. Saykally, Science 271, 59 (1996).

${ }^{11}$ R. Schwan, M. Kaufmann, D. Leicht, G. Schwaab, and M. Havenith, Phys. Chem. Chem. Phys. 18, 24063 (2016).

${ }^{12}$ R. Schwan, C. Qu, D. Mani, N. Pal, L. van der Meer, B. Redlich, C. Leforestier, J. M. Bowman, G. Schwaab, and M. Havenith, Angew. Chem. Int. Ed. 58, 2 (2019).

${ }^{13}$ N. Yang, C. H. Duong, P. J. Kelleher, A. B. McCoy, and M. A. Johnson, Science 364, 275 (2019).

${ }^{14}$ A. Gutberlet, G. Schwaab, Ö. Birer, M. Masia, A. Kaczmarek, H. Forbert, M. Havenith, and D. Marx, Science 324, 1545 (2009).

${ }^{15}$ D. Mani, R. P. De Tudela, R. Schwan, N. Pal, S. Körning, H. Forbert, B. Redlich, A. F. Van Der Meer, G. Schwaab, D. Marx, and M. Havenith, Sci. Adv. 5, eaav8179 (2019).

${ }^{16}$ M. Okumura, L. I. Yeh, J. D. Myers, and Y. T. Lee, J. Chem. Phys. 85, 2328 (1986).

${ }^{17}$ J. M. Headrick, E. G. Diken, R. S. Walters, N. I. Hammer, R. A. Christie, J. Cui, E. M. Myshakin, M. A. Duncan, M. A. Johnson, and K. D. Jordan, Science 308, 1765 (2005).

${ }^{18}$ N. Heine, M. R. Fagiani, M. Rossi, T. Wende, G. Berden, V. Blum, and K. R. Asmis, J. Am. Chem. Soc. 135, 8266 (2013).

${ }^{19}$ J. A. Fournier, C. T. Wolke, M. A. Johnson, T. T. Odbadrakh, K. D. Jordan, S. M. Kathmann, and S. S. Xantheas, J. Phys. Chem. A 119, 9425 (2015).

${ }^{20}$ C. T. Wolke, J. A. Fournier, L. C. Dzugan, M. R. Fagiani, T. T. Odbadrakh, H. Knorke, K. D. Jordan, A. B. McCoy, K. R. Asmis, and M. A. Johnson, Science 354, 1131 (2016).

${ }^{21}$ M. R. Fagiani, H. Knorke, T. K. Esser, N. Heine, C. T. Wolke, S. Gewinner, W. Schöllkopf, M. P. Gaigeot, R. Spezia, M. A. Johnson, and K. R. Asmis, Phys. Chem. Chem. Phys. 18, 26743 (2016).

${ }^{22}$ A. D. Fortes, Acta Cryst. B 74, 196 (2018).

${ }^{23}$ D. T. W. Buckingham, J. J. Neumeier, S. H. Masunaga, and Y.-K. Yu, Phys. Rev. Lett. 121, 185505 (2018).

${ }^{24}$ K. Röttger, A. Endriss, J. Ihringer, S. Doyle, and W. F. Kuhs, Acta Cryst. B 50, 644 (1994).

${ }^{25}$ K. Röttger, A. Endriss, J. Ihringer, S. Doyle, and W. F. Kuhs, Acta Cryst. B 68, 91 (2012).

${ }^{26}$ M. E. Tuckerman, D. Marx, M. L. Klein, and M. Parrinello, Science 275, 817 (1997).

${ }^{27}$ M. Benoit, D. Marx, and M. Parrinello, Nature 392, 258 (1998).

${ }^{28}$ D. Marx, M. E. Tuckerman, J. Hutter, and M. Parrinello, Nature 397, 601 (1999).

${ }^{29}$ M. E. Tuckerman and D. Marx, Phys. Rev. Lett. 86, 4946 (2001).

${ }^{30}$ M. E. Tuckerman, D. Marx, and M. Parrinello, Nature 417, 925 (2002).

${ }^{31}$ M. Benoit and D. Marx, ChemPhysChem 6, 1738 (2005).

${ }^{32}$ J. A. Morrone and R. Car, Phys. Rev. Lett. 101, 017801 (2008).

${ }^{33}$ A. Zeidler, P. S. Salmon, H. E. Fischer, J. C. Neuefeind, J. M. Simonson, and T. E. Markland, J. Phys. Condens. Matter 24, 284126 (2012).

${ }^{34}$ M. Ceriotti, J. Cuny, M. Parrinello, and D. E. Manolopoulos, Proc. Natl. Acad. Sci. USA 110, 15591 (2013).

${ }^{35}$ L. Wang, M. Ceriotti, and T. E. Markland, J. Chem. Phys. 141, 104502 (2014).

${ }^{36}$ C. Schran, O. Marsalek, and T. E. Markland, Chem. Phys. Lett. 678, 289 (2017).

${ }^{37} \mathrm{~S}$. Habershon, T. E. Markland, and D. E. Manolopoulos, J. Chem. Phys. 131, 024501 (2009).

${ }^{38}$ X.-Z. Li, B. Walker, and A. Michaelides, Proc. Natl. Acad. Sci. USA 108, 6369 (2011).

${ }^{39}$ F. Uhl, D. Marx, and M. Ceriotti, J. Chem. Phys. 145, 054101 (2016).

${ }^{40}$ C. Schran, F. Brieuc, and D. Marx, J. Chem. Theory Comput. 14, 5068 (2018). 
${ }^{41}$ M. Ceriotti, J. More, and D. E. Manolopoulos, Comput. Phys. Commun. 185, 1019 (2014).

42 "CP2K, freely available at the URL https://www.cp2k.org, released under GPL license," (2019).

${ }^{43}$ J. Hutter, M. Iannuzzi, F. Schiffmann, and J. Vandevondele, Wiley Interdiscip. Rev. Comput. Mol. Sci. 4, 15 (2014).

${ }^{44}$ C. Schran, J. Behler, and D. Marx, arXiv:1908.08734 [physics.chem-ph] (2019).

${ }^{45}$ S. Plimpton, J. Comput. Phys. 117, 1 (1995).

${ }^{46}$ R. Ramírez and C. P. Herrero, J. Chem. Phys. 133, 144511 (2010).

${ }^{47}$ C. P. Herrero and R. Ramírez, J. Chem. Phys. 134, 094510 (2011).

${ }^{48}$ C. P. Herrero and R. Ramírez, Phys. Rev. B 84, 224112 (2011).

${ }^{49}$ S. Habershon and D. E. Manolopoulos, Phys. Chem. Chem. Phys. 13, 19714 (2011).

${ }^{50}$ T. E. Markland and B. J. Berne, Proc. Natl. Acad. Sci. USA 109, 7988 (2012).

${ }^{51}$ V. Kapil, A. Cuzzocrea, and M. Ceriotti, J. Phys. Chem. B 122, 6048 (2018).

${ }^{52}$ V. Babin and F. Paesani, Chem. Phys. Lett. 580, 1 (2013).

${ }^{53}$ M. Ceriotti and D. E. Manolopoulos, Phys. Rev. Lett. 109, 100604 (2012).

${ }^{54}$ C. Drechsel-Grau and D. Marx, Phys. Rev. Lett. 112, 148302 (2014).

${ }^{55}$ C. Drechsel-Grau and D. Marx, Angew. Chem. Int. Ed. 53, 10937 (2014).

${ }^{56}$ J. VandeVondele, M. Krack, F. Mohamed, M. Parrinello, T. Chassaing, and J. Hutter, Comput. Phys. Commun. 167, 103 (2005).

${ }^{57}$ B. Hammer, L. B. Hansen, and J. K. Nørskov, Phys. Rev. B 59, 7413 (1999)
${ }^{58}$ S. Grimme, J. Antony, S. Ehrlich, and H. Krieg, J. Chem. Phys. 132, 154104 (2010).

${ }^{59}$ S. Goedecker, M. Teter, and J. Hutter, Phys. Rev. B 54, 1703 (1996).

${ }^{60}$ T. Morawietz and J. Behler, J. Phys. Chem. A 117, 7356 (2013).

${ }^{61}$ K. Forster-Tonigold and A. Groß, J. Chem. Phys. 141, 064501 (2014).

${ }^{62}$ T. Morawietz, A. Singraber, C. Dellago, and J. Behler, Proc. Natl. Acad. Sci. USA 113, 8368 (2016).

${ }^{63}$ A. Luzar and D. Chandler, Phys. Rev. Lett. 76, 928 (1996).

${ }^{64}$ R. Kumar, J. R. Schmidt, and J. L. Skinner, J. Chem. Phys. 126, 204107 (2007).

${ }^{65}$ U. Weiss, Quantum Dissipative Systems, 4th ed. (World Scientific, Singapore, 2012).

${ }^{66}$ M. Benoit, A. H. Romero, and D. Marx, Phys. Rev. Lett. 89, 145501 (2002).

${ }^{67}$ K. Stokely, M. G. Mazza, H. E. Stanley, and G. Franzese, Proc. Natl. Acad. Sci. USA 107, 1301 (2008).

${ }^{68}$ J. M. Guevara-Vela, E. Romero-Montalvo, V. A. Mora Gómez, R. Chávez-Calvillo, M. García-Revilla, E. Francisco, Á. M. Pendás, and T. Rocha-Rinza, Phys. Chem. Chem. Phys. 18, 19557 (2016).

${ }^{69}$ A. Novak, in Struct. Bond. Vol. 18 Large Mol. (Springer, Berlin, Heidelberg, 1974) pp. 177-216.

${ }^{70}$ D. A. Schmidt and K. Miki, J. Phys. Chem. A 111, 10119 (2007).

${ }^{71}$ Y. A. Galkina, N. A. Kryuchkova, M. A. Vershinin, and B. A. Kolesov, J. Struct. Chem. 58, 911 (2017).

${ }^{72}$ L. Walewski, H. Forbert, and D. Marx, Mol. Phys. 111, 2555 (2013).

${ }^{73}$ L. Walewski, H. Forbert, and D. Marx, J. Chem. Phys. 140, 144305 (2014). 